\title{
Summary of Glaucoma Diagnostic Testing Accuracy: An Evidence-Based Meta-Analysis
}

\author{
Saad Ahmed ${ }^{\mathrm{a}}$, Zainab Khan ${ }^{\mathrm{b}}$, Francie $\mathrm{Si}^{\mathrm{a}}$, Alex Mao ${ }^{\mathrm{a}}$, Irene Pan ${ }^{\mathrm{a}}$, Fatemeh Yazdic, \\ Alexander Tsertsvadze ${ }^{\mathrm{c}}$, Cindy Hutnik ${ }^{\mathrm{a}}$, David Moher ${ }^{\mathrm{c}}$, David Tingey ${ }^{\mathrm{a}}$, \\ Graham E. Trope ${ }^{\mathrm{d}}$, Karim F. Damji ${ }^{\mathrm{e}}$, Jean-Eric Tarride ${ }^{\mathrm{f}}$, Ron Goeree ${ }^{\mathrm{f}}$, William Hodge ${ }^{\mathrm{a}, \mathrm{g}, \mathrm{h}}$
}

\begin{abstract}
Background: New glaucoma diagnostic technologies are penetrating clinical care and are changing rapidly. Having a systematic review of these technologies will help clinicians and decision makers and help identify gaps that need to be addressed. This systematic review studied five glaucoma technologies compared to the gold standard of white on white perimetry for glaucoma detection.
\end{abstract}

Methods: OVID ${ }^{\circledR}$ interface: MEDLINE ${ }^{\circledR}$ (In-Process \& Other NonIndexed Citations), EMBASE ${ }^{\circledR}$, BIOSIS Previews ${ }^{\circledR}$, CINAHL $^{\circledR}$, PubMed, and the Cochrane Library were searched. A gray literature search was also performed. A technical expert panel, information specialists, systematic review method experts and biostatisticians were used. A PRISMA flow diagram was created and a random effect metaanalysis was performed.

Results: A total of 2,474 articles were screened. The greatest accuracy was found with frequency doubling technology (FDT) (diagnostic odds ratio (DOR): 57.7 ) followed by blue on yellow perimetry (DOR: 46.7), optical coherence tomography (OCT) (DOR: 41.8), GDx (DOR: 32.4 ) and Heidelberg retina tomography (HRT) (DOR: 17.8). Of greatest concern is that tests for heterogeneity were all above $50 \%$, indicating that cutoffs used in these newer technologies were all very varied and not uniform across studies.

Manuscript accepted for publication July 13, 2016

avey Eye Institute, University of Western Ontario, London, ON, Canada

bFaculty of Medicine, Queen's University, Kingston, ON, Canada

'Ottawa Hospital Research Institute, University of Ottawa, Ottawa, ON, Canada

dDepartment of Ophthalmology and Visual Sciences, University of Toronto, Toronto, ON, Canada

eDepartment of Ophthalmology, University of Alberta, Edmonton, AB, Canada

PProgram for the Assessment of Technology and Health (PATH), and Department of Clinical Epidemiology and Biostatistics, McMaster University, Hamilton, ON, Canada

gDepartment of Epidemiology and Biostatistics, University of Western Ontario, London, ON, Canada

${ }^{\mathrm{h}}$ Corresponding Author: William Hodge, Ivey Eye Institute, University of Western Ontario, 268 Grosvenor St, London, ON N6A 4V2, Canada.

Email: William.hodge@sjhc.london.on.ca

doi: http://dx.doi.org/10.14740/jocmr2643w
Conclusions: Glaucoma content experts need to establish uniform cutoffs for these newer technologies, so that studies that compare these technologies can be interpreted more uniformly. Nevertheless, synthesized data at this time demonstrate that amongst the newest technologies, OCT has the highest glaucoma diagnostic accuracy followed by GDx and then HRT.

Keywords: Glaucoma; Diagnostic techniques; Diagnostic accuracies; Systematic review; Meta-analysis

\section{Introduction}

With the aging Western populations, special attention needs to be directed to the research of age-related eye diseases. Glaucoma is one of the leading causes of visual loss and blindness in the working age population in industrialized countries [14]. In most Western nations, aside from age-related macular degeneration, glaucoma is the next most common cause of permanent vision loss in persons aged 40 years or older [5]. Along with diabetic retinopathy, glaucoma is also one of the most important diseases affecting the visual system in the working age population [3-5]. Finally, the extensive and intensive work needed to make the diagnosis, the multiple management changes needed over the course of the disease, and the life-long follow-up required arguably make this condition the most important ocular disease from the health services standpoint.

Previous research has shown that early diagnosis and treatment for glaucoma reduces the rate of disease progression and improves the patients' quality of life [6]. The current "gold standards" for glaucoma diagnosis are optic disc assessment (monitors structural change) and standard achromatic white on white perimetry (monitors functional change) [7].

Several diagnostic technologies were recently developed for early glaucoma diagnosis and progression.

1) Heidelberg retina tomography (HRT) $[8,9]$ uses a 675 $\mathrm{nm}$ frequency diode laser beam to produce a scanning laser ophthalmoscopic image. The laser light scans the retina in 24 ms sequential scans, starting above the retinal surface, and then capturing parallel images at increasing depths. The stacks of images are combined to create a three-dimensional (3D) 
topographic image of the retina.

2) Optical coherence tomography (OCT) [10-13] provides high-resolution cross-sectional imaging of ocular tissues (retina, optic nerve, and anterior segment). The technique is analogous to ultrasound except that light instead of sound waves is used. OCTs operate on the principle of indirect interferometry. Low coherence infrared light is directed to the tissue being imaged. An interferometer (beam splitter) is used to split the light. Half of the light goes to the target tissue and the other half goes into a reference arm (typically a mirror).

3) Scanning laser polarimetry (GDx variable corneal compensator (VCC) access) [14-16] uses a near infrared laser beam to measure the thickness of the retinal nerve fiber layer. This technology is based on the optical attribute of birefringence (parallel arrangement of the microtubules within the axons). The nerve fiber layer birefringence causes the polarized laser beam to split into two parallel rays and creates a phase shift between them; one is more distorted than the other.

4) Frequency doubling technology (FDT) is a form of perimetry developed to detect ganglion cell death in early glaucomatous damage. Studies $[17,18]$ hypothesize that a subset of $\mathrm{M}$ cells, called "non-linear" $\mathrm{M}$ cells, are usually the first to die during glaucomatous damage. This subset of cells is thought to be responsible for the transmission of signals related to the frequency doubling illusion.

5) Blue on yellow automated perimetry [19-22] isolates and measures blue-yellow ganglion cell function with a 440 $\mathrm{nm}$ stimulus. Blue-yellow ganglion cells are believed to be one of the first cell types damaged in glaucoma and thus seem to be important in early glaucoma detection.

The diagnostic instruments listed above have been reported to detect glaucomatous damage. There is some evidence that earlier damage can be detected with HRT than with clinical examination or fundus photography [23, 24]. However, there has not been a comprehensive report on its accuracy compared to that of the reference standard. The goal of this systematic review is to summarize the diagnostic accuracy of recently developed glaucoma diagnostic technologies compared to the currently available gold standard, white on white perimetry.

\section{Materials and Methods}

\section{Overview of evidence report}

The research synthesis for glaucoma diagnostic accuracies was based on a rigorous systematic review of all published reports. Together with content experts, methodologists, information specialists, and statisticians, the evidence review staff identified specific issues and approaches necessary for limiting bias in the review.

A technical expert panel (TEP) identified the keywords to be used in the literature search. The methodological quality and generalizability (internal and external validity) of the included studies were critically appraised and summarized, as was individual study results.

\section{Sources of data}

The search strategy for this project was comprehensive and was tailored to achieve the highest possible recall of relevant studies. An electronic search strategy was developed by an information specialist in consultation with four clinical content experts in glaucoma diagnostics. Searches were restricted from 1993 onward to exclude obsolete diagnostic options. The search end date was February 2015. Any study providing information on the sensitivity, specificity, likelihood ratio, receiver-operating characteristic (ROC) curves, or diagnostic odds ratios (DORs) of a glaucoma diagnostic test was included. Specific search terms tailored for diagnostic testing have been empirically studied and were used [25, 26]. Published and unpublished human studies of any design were considered. The reference standard used was standard achromatic white on white perimetry.

The following bibliographic databases were searched through the OVID ${ }^{\circledR}$ interface: MEDLINE ${ }^{\circledR}$ (In-Process \& Other Non-Indexed Citations), EMBASE ${ }^{\circledR}$, BIOSIS Previews ${ }^{\circledR}$, CINAHL $^{\circledR}$, PubMed, and the Cochrane Library. Controlled vocabulary and keywords used in the search included terms for glaucoma diagnostic testing including the specific evaluative tests and the gold standard test already mentioned in these methods. Methodological filters were applied to limit retrieval to relevant clinical methodologies applicable to diagnostic testing.

The gray literature was searched including websites of health technology assessment and related agencies, professional associations, and other specialized databases. Google $\mathrm{TM}^{\mathrm{TM}}$ and other Internet search engines were used to search for additional web-based materials and information. These searches were supplemented by hand searching the bibliographies and abstracts of key papers and conference proceedings, and through contacts with appropriate experts and agencies. These include the Association for Research in Vision and Ophthalmology, the American Academy of Ophthalmology, the European Ophthalmology Society and The Canadian Ophthalmology Association.

\section{Inclusion criteria}

Any study evaluating testing outcomes for open angle glaucoma diagnosis was considered for inclusion in our review to maximize interpretability and generalizability. A standardized and accepted approach for gold standard assessment in study populations (white on white automated perimetry) was required for inclusion. We originally were to include optic disc photographs as a parallel gold standard but there were so few studies that used this gold standard that it was impractical to use this as a reference standard. Only studies with a minimum of 20 adult patients were included. Any geographic study type (single vs. multi-center) was included as was any sampling strategy. Patients from all ethnic background and studies conducted in any country were included. Patients from all study bases were included (population or clinic based samples). All patients included were 18 years of 


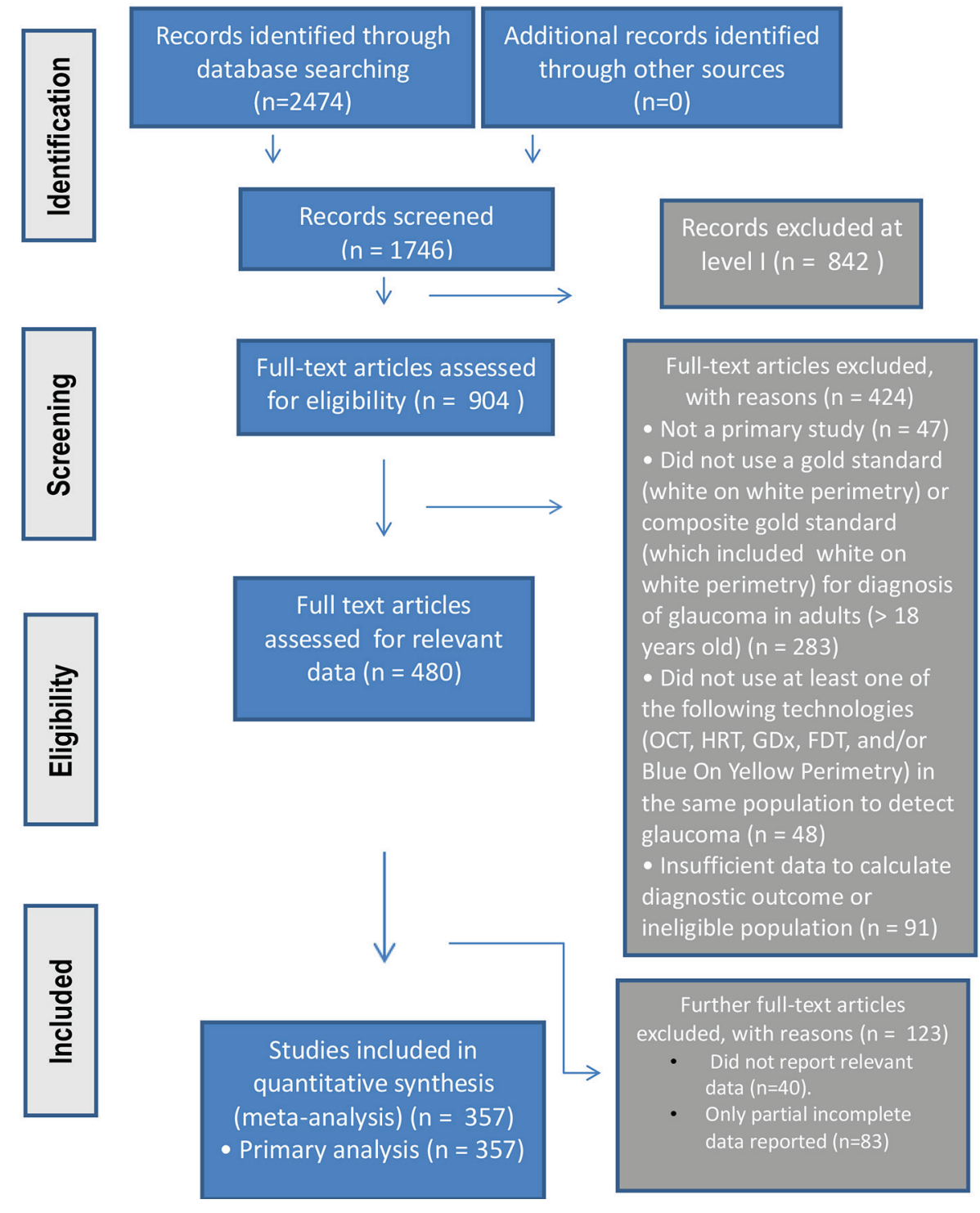

Figure 1. The PRISMA study process summarized in flowchart format.

age or older.

\section{Study selection}

Specific screening questions were drafted for all levels of relevance assessment, and following an appropriate calibration exercise, screening was performed by two independent reviewers. All records were uploaded to a software program (EPPI reviewer) to help manage the review. All records retrieved through searches underwent broad screening initially (level I) using titles, keywords and abstracts, if available. All records tagged by one or more reviewers as potentially relevant or "can't tell" (including reviews, statements and original studies) were screened for inclusion at the next level (full-text relevance, level II) using stricter a priori eligibility criteria. Reference lists of reviews that were thought to be relevant were also checked for potentially relevant publications.
At levels where consensus was required, and when disagreements occurred, consensus was achieved through discussion. When agreement could not be achieved, third party adjudication was used to break the impasse. Reasons for exclusion of studies were documented and reported in the review. The study followed the PRISMA statement methodology and a PRISMA flow diagram was used to report the flow of records throughout the review [27].

\section{Data extraction}

An electronic data abstraction form was developed a priori and tailored specifically for this review. Data collected included study identification data (country, language, and citation), research methods data (study design, sites, and inclusion/ exclusion criteria), population baseline variables (sample size 
Table 1. Overall Assessment of Study Quality, Accuracy and Generalizability (QUADAS)

\begin{tabular}{|c|c|c|c|}
\hline Items & $\%$ assessed as yes & $\%$ assessed as no & $\%$ assessed as unclear \\
\hline $\begin{array}{l}\text { 1. Was the spectrum of patients representative of the patients who will } \\
\text { receive the test in practice? }\end{array}$ & 44 & 15 & 41 \\
\hline $\begin{array}{l}\text { 3. Is the reference standard likely to correctly classify the target } \\
\text { condition? }\end{array}$ & 93 & 4 & 3 \\
\hline $\begin{array}{l}\text { 4. Is the time period between reference standard and index test short } \\
\text { enough to be reasonably sure that the target condition did not change } \\
\text { between the two tests? }\end{array}$ & 93 & 0 & 7 \\
\hline $\begin{array}{l}\text { 5. Did the whole sample or a random selection of the sample, receive } \\
\text { verification using a reference standard of diagnosis? }\end{array}$ & 73 & 3 & 24 \\
\hline $\begin{array}{l}\text { 6. Did patients receive the same reference standard } \\
\text { regardless of the index test result? }\end{array}$ & 99 & 0 & 1 \\
\hline $\begin{array}{l}\text { 9. Was the execution of the reference standard described in sufficient } \\
\text { detail to permit its replication? }\end{array}$ & 95 & 2 & 3 \\
\hline $\begin{array}{l}\text { 10. Were the index test results interpreted without knowledge of the } \\
\text { results of the reference standard? }\end{array}$ & 82 & 3 & 15 \\
\hline $\begin{array}{l}\text { 11. Were the reference standard results interpreted without knowledge of } \\
\text { the results of the index test? }\end{array}$ & 22 & 6 & 72 \\
\hline $\begin{array}{l}\text { 12. Were the same clinical data available when test results were } \\
\text { interpreted as would be available when the test is used in practice? }\end{array}$ & 26 & 2 & 72 \\
\hline 13. Were un-interpretable/ intermediate test results reported? & 69 & 9 & 22 \\
\hline
\end{tabular}

complete and enrolled, mean age, gender, and race), clinical variables (visual acuity, IOP, C/D ratio, and central cornea thickness), white on white VF variables (mean defect, and corrected pattern standard deviation) and index technology variables (cutoffs and methods used). Following a calibration exercise on a random sample of studies, one data abstractor independently abstracted data, and a second reviewer then independently verified the data. The data form was designed to capture all relevant data. For example, study population characteristics as well as characteristics of the study itself that might identify potential sources of bias were included. In studies where multiple sensitivities and specificities were provided, we always chose the value for the highest sensitivity reported and then chose the corresponding specificity with it. If data were reported as normal vs. "mild", "moderate", and "severe" glaucoma, we always chose to report "mild" as this gives us the most important diagnostic information (e.g. "advanced" glaucoma is much easier to diagnose and hence the test is less useful).

\section{Study quality assessment}

The QUADAS [28, 29] 14-item assessment tool was used to assess bias (nine items), variability (affecting generalizability) (two items) and to some extent, the quality of reporting (three items). Each item is to be scored "yes," "no," or "unclear."

\section{Methods for synthesizing findings - data analysis}

Random-effects meta-analysis was performed for each diagnostic instrument where possible and stratified by the type of summary statistics available from eligible studies. For most aspects of meta-analysis, it is preferred that the diagnostic threshold be the same across different studies before the parameters are pooled for analysis. However, ROC curves can be pooled for different diagnostic thresholds if variance is provided. The parameters of interest included sensitivities, specificities, likelihood ratios, ROC curves, and DORs. The DOR is a parameter that combines sensitivity and specificity and ranges from 0 to infinity with higher values indicating greater test performance. Because DORs combine the results of both sensitivity and specificity, we used this as our primary outcome.

\section{Summary of synthesized deliverables}

We organized the review to answer both primary and second- 
Table 2. Accuracy of Diagnostic Technologies (Listed By Number of Studies Reviewed)

\begin{tabular}{|c|c|c|c|c|c|}
\hline & \multicolumn{2}{|c|}{ \# Studies } & \multicolumn{3}{|c|}{ Pooled results } \\
\hline & Sensitivity & Specificity & Sensitivity & Specificity & Diagnostic odds ratio \\
\hline \multicolumn{6}{|l|}{ Primary analysis } \\
\hline GDx (FCC and VCC) & 103 & 103 & $84.5(81.7-87.0)$ & $85.6(82.9-87.9)$ & $32.4(25.6-40.9)$ \\
\hline OCT (all types) & 84 & 84 & $83.2(80.7-85.4)$ & $89.4(87.5-91.0)$ & $41.8(33.5-52.0)$ \\
\hline \multicolumn{6}{|l|}{ Secondary analysis } \\
\hline HRT I and II & 102 & 102 & $77.8(75.3-80.0)$ & $84.5(82.2-86.6)$ & $19.1(15.7-23.1)$ \\
\hline HRT III & 30 & 30 & $81.9(77.3-85.7)$ & $76.3(71.7-80.4)$ & $14.6(11.4-18.6)$ \\
\hline GDx-VCC & 98 & 98 & $84.9(82.1-87.3)$ & $85.7(83.0-88.0)$ & $33.7(26.4-42.9)$ \\
\hline OCT-spectralis & 1 & 1 & \multicolumn{3}{|c|}{ Only one study - no pooled results } \\
\hline FDT & 57 & 57 & $84.7(79.1-89.0)$ & $91.2(88.2-93.5)$ & $57.7(35.8-92.9)$ \\
\hline Blue on yellow & 5 & 6 & $87.2(67.2-95.8)$ & $83.2(68.4-91.9)$ & $46.7(7.58-288.2)$ \\
\hline
\end{tabular}

ary research questions.

The primary research question addressed the diagnostic accuracies of five index technologies for glaucoma screening: 1) confocal scanning laser ophthalmoscopy (divided into HRT I-II and HRT III); 2) OCT; 3) scanning laser polarimetry; 4) blue on yellow automated perimetry; and 5) FDT.

A variety of models are available for each index technology. Secondary research questions that were addressed were how the models of each index technology compare against gold standard for glaucoma diagnosis. The specific secondary technologies studied were as the following: 1) FDT (no subdivisions). 2) OCT (time domain, spectral domain, and OCT spectralis by Heidelberg). 3) Confocal scanning laser ophthalmoscopy (HRT I and HRT II; HRT III). 4) Blue on yellow automated perimetry (no subdivisions). Although Swedish interactive threshold algorithm and full threshold are two available options, most studies included in this review did not indicate which of the two programs were utilized. 5) Scanning laser polarimetry (GDx VCC; GDx fixed corneal compensator (FCC)).

\section{Results}

The study process was summarized in the PRISMA flowchart (Fig. 1).

\section{Study quality}

Table 1 summarizes the study quality and generalizability based on the QUADAS questions. Most of the accuracy/quality indicators rated the studies as very good to excellent. The generalizability indicators were moderate to good.

\section{Study characteristics}

Overall 357 studies were included in the analysis. There were $53.8 \%$ female and $46.2 \%$ male (reported in 256 studies). The mean age of the participants was 58.4 years, ranging from 41.0 to 69.7 years. Overall $42.9 \%$ of the participants were Caucasian. All studies reviewed were studies of glaucoma diagnosis. No studies of progression were used.

\section{Diagnostic accuracy of technology}

Table 2 summarizes the sensitivity, specificity and DOR for each of our primary and secondary outcomes. As can be seen from the results, amongst the primary outcomes, the highest DOR is from FDT followed by blue on yellow perimetry (but with the fewest studies and widest confidence intervals). Hence, the "older" new technologies had the highest DORs. Amongst the "newer" new technologies, OCT had the highest DOR followed by scanning laser (GDx), and HRT had the lowest accuracy.

When we stratified our technologies into our secondary outcomes, the results are similar but there are a few additional important points. First, the spectral domain OCT performed much better than the time domain OCT. Secondly, the GDx VCC performed much better than the GDx FCC. Finally, the HRT III has not shown an increase in diagnostic performance over HRT I and II. We also tried to stratify the data by study size, separating clinical based studies from larger populationbased epidemiology studies. Unfortunately, only three popula- 
Table 3. Sub-Analysis of Accuracy of Diagnostic Technology (Based on Outlier Removal, Funding Source, Age, Race and Gender)

\begin{tabular}{|c|c|c|c|c|c|c|c|c|c|c|c|}
\hline DORs & Overall & $\begin{array}{l}\text { Overall less } \\
\text { outliers }\end{array}$ & $\begin{array}{l}\text { Peer } \\
\text { review }\end{array}$ & Industry & $\begin{array}{l}\text { Age } \\
>55\end{array}$ & $\begin{array}{l}\text { Age } \\
<55\end{array}$ & $\begin{array}{l}\text { Cauc } \\
>50 \%\end{array}$ & $\begin{array}{l}\text { Cauc } \\
<50 \%\end{array}$ & $\begin{array}{l}\text { Fem } \\
>\mathbf{5 0} \%\end{array}$ & $\begin{array}{l}\text { Fem } \\
<50 \%\end{array}$ & AUC \\
\hline HRT & 17.82 & 18.18 & 16.94 & 18.56 & 17.56 & 19.99 & 19 & 13.99 & 17.64 & 18.62 & 0.839 \\
\hline OCT & 41.76 & 43.36 & 39.29 & 43.62 & 41.26 & 47.11 & 43.2 & 38.92 & 49.14 & 25.94 & 0.901 \\
\hline FDT & 57.7 & 60.96 & 57.29 & 58.03 & 56.01 & 61.18 & 57.53 & 67.71 & 57.53 & 45.44 & 0.893 \\
\hline HRT III & 14.56 & 15.17 & 24.5 & 13.1 & 13.82 & NA & 16.89 & 10.72 & 15.31 & 13.07 & 0.829 \\
\hline GDx VCC & 33.65 & 31.27 & 35.61 & 32.06 & 32.97 & 41.41 & 34.54 & 24.5 & 35.09 & 31.61 & 0.882 \\
\hline GDx FCC & 17.46 & 17.46 & NA & 17.46 & 17.46 & NA & 15.03 & NA & 15.03 & NA & 0.859 \\
\hline OCT-T & 38.73 & 38 & 34.15 & 42.92 & 38.66 & 50.13 & 41.28 & 31.63 & 45.34 & 23.91 & 0.898 \\
\hline
\end{tabular}

tion-based studies were found and all studied FDT only compared to gold standard. Hence, this stratified meta-analysis was not possible.

\section{Sub-analyses}

Table 3 demonstrates all of the sub-analyses performed. In column 2 of the table, we recalculated the DORs after removing outlier studies (based on the Begg's test). As can be seen, there was little difference in the results when these outlier studies were removed. Next we looked at DORs from peer reviewed vs Industry funded studies. In this analysis we postulated that there may be higher DORs from industry funded studies over peer reviewed studies as there may be a bias to obtain good results for retail purposes. However, the results did not show this. While some technologies had a small increase in DORs amongst industry funded studies, others showed higher DORs from peer reviewed funding. Overall there was no significant pattern of increased DORs from industry vs. peer reviewed funded studies. We also looked at whether age had an influence on the diagnostic accuracy of the technologies. We found that when we divided studies into those where mean age was over 55 vs. under 55, the under 55 group did better in almost every analysis. When we divided the studies based on predominance of Caucasians as the majority race, we found that almost all technologies performed better amongst Caucasians vs. nonCaucasians. Finally, most of the technologies had higher ac-

Table 4. Heterogeneity of Diagnostic Technology

\begin{tabular}{|llll}
\hline Technology & Sensitivity $\mathbf{I}^{2}$ & Specificity $\mathbf{I}^{2}$ & Diagnostic odds ratio $\mathbf{I}^{\mathbf{2}}$ \\
\hline Primary outcome & & & \\
\hline HRT & 68.7 & 86.3 & 71.4 \\
GDx & 86.2 & 85.3 & 72.5 \\
OCT & 75.3 & 73.7 & 69.5 \\
FDT & 97.5 & 94.7 & 94.3 \\
Blue on yellow & 79.8 & 79.1 & 84.4 \\
Secondary outcomes & & & \\
\hline HRT I and II & 78.7 & 87.3 & 81.4 \\
HRT III & 60.9 & 85.3 & 62.1 \\
Scanning laser VCC & 86.6 & 85.2 & 74.5 \\
\hline Scanning laser FCC & 85.5 & 83.3 & 84.0 \\
Time domain OCT & 76.7 & 75.6 & 70.5 \\
Spectral domain OCT & 73.0 & 63.7 & 65.9 \\
\hline FDT & 97.5 & 94.7 & 94.3 \\
Blue on yellow & 79.8 & 79.1 & 84.4 \\
\hline
\end{tabular}


Table 5. Number of Cutoffs Used Stratified by Technology

\begin{tabular}{ll}
\hline Technology & Number of methods/cutoffs encountered \\
\hline OCT & 52 \\
GDx & 66 \\
Blue on yellow & 4 \\
FDT & 52 \\
HRT I and II & 43 \\
HRT III & 6 \\
\hline
\end{tabular}

curacy amongst women over men.

\section{Heterogeneity}

Table 4 summarizes $\mathrm{I}^{2}$ for each technology stratified by sensitivity, specificity and DOR. This statistic is a statistic of homogeneity. In other words, it tells us how similar the studies are that are compared. Studies that are similar tend to pool results with the same cutoff and their technologies have a significant amount of agreement between users. Their $\mathrm{I}^{2}$ values are close to zero or are at least less than 50 . An $\mathrm{I}^{2}$ above 50 and even close to 100 as is true for these technologies demonstrates a large amount of heterogeneity.

The reason for such a large amount of heterogeneity was explored and can be summarized by simply tabulating the total number of different methods and cutoffs used to assess the technology in question. As an example, for OCT it is possible to assess any clock hour, quadrant or average nerve fiber layer thickness to assess the detection of glaucoma. Furthermore, what cutoff is used to accept the diagnosis of glaucoma can be different from study to study even for the same assessment (e.g. average nerve fiber layer thickness). The total number of methods and cutoffs used in our systematic review are summarized in Table 5.

\section{Discussion}

The QUADAS tool reveals that the overall quality within individual studies was very good (almost $75 \%$ of individual items were positive). However, one of the most important conclusions from this study is that the heterogeneity of these new glaucoma tests between studies is very high. This is disappointing given that there were 2,474 articles screened which makes the review very comprehensive and generalizable. When methods of outcome analysis, as well as cutoffs are this varied, it is very difficult to be certain about the accuracy and effectiveness of a test. It is our conclusion that the content experts of this field need to determine cutoffs and methods that can be agreed upon to make these tests more uniform. At that point, the most accurate comparisons between technologies will be made. A pervasive challenge with all of these diagnostic tools is the heavy marketing influence that can add further inconsistencies and heterogeneity in their utilization.

Nevertheless, the synthesis of existing data, though varied and heterogeneous, does give us some interesting information that is useful. First, of the five main technologies tested, the highest DOR, mainly based on specificity occurred with FDT. The next highest DOR belonged to blue on yellow perimetry based on a high sensitivity. Both of these "new" diagnostic tests have been with us for over a decade but are not in widespread use. Of the newer technologies, OCT had the highest accuracy (especially spectral domain OCT) followed by GDx. GDx VCC had a higher accuracy than GDx FCC. Finally, HRT was the least accurate and accuracy parameters for HRT I and II were not very different than HRT III. These are interesting results given that the HRT was heavily marketed for utilization in the management of glaucoma being the first technology to have a reasonable normative database as well as progression monitoring software.

When we stratified the main technologies into several secondary technologies, other interesting information was revealed. The stratus OCT was much more accurate, especially more specific than the time domain OCT. This is not surprising as each new generation of OCT has offered substantially more resolution than its predecessor. Compared to HRT, upgrades in the OCT technology have been more frequent but progression assessment has not been possible to date.

The scanning laser (GDx) with a VCC was much more sensitive and specific than the GDx with an FCC. Although GDx was one of the first to be introduced, it has lagged behind the other two in terms of general adoption into clinical practice and seems to be less popular today.

Sub-analyses showed that outliers did not influence our results. This means that the results found are robust to a few studies that may "pull" the means disproportionately. Our results were not influenced by funding source, industry vs. peer reviewed. Our results were more accurate amongst younger patients, Caucasians and women.

Synthesis studies of these new technologies are made especially challenging by the rapid pace of software changes for all of the methods. This makes assessing the technologies difficult as technology changes can occur even before robust evidence syntheses can be completed with evidence-based methodology.

In summary, work needs to be done by glaucoma content experts to create a more homogenous consensus regarding how to utilize the new technologies and to agree on cutoffs. At that point, it will be possible to determine if our conclusions regarding the relative accuracy of these newer technologies remains valid. However, synthesis of results at this point in time indicates that amongst the newer glaucoma diagnostic technologies, OCT is the most accurate followed by GDx and then HRT.

\section{Acknowledgments}

We give thanks to the funding agency of CIHR for making the study possible. We thank Dr. David Hill for helping to obtain the funding. We also thank Becky Skidmore for literature search, Sophia Tsouros and Jeff Brunton with the use of the software EPPI. 


\section{Financial Support}

Canadian Institutes of Health Research (CIHR) Knowledge Synthesis Grant \#KRS 91789.

\section{Conflicts of Interest}

No conflicting relationship exists for any author.

\section{Author Contributions}

S. Ahmed: conception, data acquisition and drafting manuscript; Z. Khan: conception, data acquisition and drafting manuscript; F. Si: team coordination and administration, conception, data acquisition, manuscript revising, paper preparation and submission; A. Mao: conception and data acquisition; I. Pan: conception and data acquisition; F. Yazdi: conception, data acquisition and analysis; A. Tsertsvadze: conception, data analysis and revising manuscript; C. Hutnik: conception, data acquisition and interpretation, revising manuscript; D. Moher: help with obtaining funding and conception; D. Tingey: conception and revising manuscript; G. Trope: conception and revising manuscript; K. Damji: conception and revising manuscript; J. E. Tarride: conception and revising manuscript; R. Goeree: help with obtaining funding and revising manuscript; W. Hodge (guarantor of the paper): obtaining funding, supervision, conception, design; data acquisition, analysis and interpretation, drafting article and revising.

\section{References}

1. Quigley HA. Number of people with glaucoma worldwide. Br J Ophthalmol. 1996;80(5):389-393.

2. Quigley HA, Vitale S. Models of open-angle glaucoma prevalence and incidence in the United States. Invest Ophthalmol Vis Sci. 1997;38(1):83-91.

3. Tuck MW, Crick RP. The projected increase in glaucoma due to an ageing population. Ophthalmic Physiol Opt. 2003;23(2):175-179.

4. Munier A, Gunning T, Kenny D, O'Keefe M. Causes of blindness in the adult population of the Republic of Ireland. Br J Ophthalmol. 1998;82(6):630-633.

5. Buhrmann R, Hodge W, Beardmore J, et al. Foundations for a Canadian Vision Health Strategy: Towards Preventing Avoidable Blindness and Promoting Vision Health. Ottawa: University of Ottawa, The Ottawa Hospital General Campus: Eye Institute, 2007.

6. Quigley HA, Katz J, Derick RJ, Gilbert D, Sommer A. An evaluation of optic disc and nerve fiber layer examinations in monitoring progression of early glaucoma damage. Ophthalmology. 1992;99(1):19-28.

7. Medeiros FA, Zangwill LM, Bowd C, Sample PA, Weinreb $\mathrm{RN}$. Use of progressive glaucomatous optic disk change as the reference standard for evaluation of diagnostic tests in glaucoma. Am J Ophthalmol. 2005;139(6):1010-1018.
8. Wollstein G, Garway-Heath DF, Hitchings RA. Identification of early glaucoma cases with the scanning laser ophthalmoscope. Ophthalmology. 1998;105(8):15571563.

9. Kamal DS, Viswanathan AC, Garway-Heath DF, Hitchings RA, Poinoosawmy D, Bunce C. Detection of optic disc change with the Heidelberg retina tomograph before confirmed visual field change in ocular hypertensives converting to early glaucoma. $\mathrm{Br} \mathrm{J}$ Ophthalmol. 1999;83(3):290-294.

10. Bowd C, Weinreb RN, Williams JM, Zangwill LM. The retinal nerve fiber layer thickness in ocular hypertensive, normal, and glaucomatous eyes with optical coherence tomography. Arch Ophthalmol. 2000;118(1):22-26.

11. Schuman JS, Hee MR, Arya AV, Pedut-Kloizman T, Puliafito CA, Fujimoto JG, Swanson EA. Optical coherence tomography: a new tool for glaucoma diagnosis. Curr Opin Ophthalmol. 1995;6(2):89-95.

12. Hrynchak P, Simpson T. Optical coherence tomography: an introduction to the technique and its use. Optom Vis Sci. 2000;77(7):347-356.

13. Kee C, Cho C. Evaluation of retinal nerve fiber layer thickness in the area of apparently normal hemifield in glaucomatous eyes with optical coherence tomography. J Glaucoma. 2003;12(3):250-254.

14. Yamada N, Chen PP, Mills RP, Leen MM, Stamper RL, Lieberman MF, Xu L, et al. Glaucoma screening using the scanning laser polarimeter. J Glaucoma. 2000;9(3):254261.

15. Choplin NT, Lundy DC. The sensitivity and specificity of scanning laser polarimetry in the detection of glaucoma in a clinical setting. Ophthalmology. 2001;108(5):899904.

16. Weinreb RN, Zangwill L, Berry CC, Bathija R, Sample PA. Detection of glaucoma with scanning laser polarimetry. Arch Ophthalmol. 1998;116(12):1583-1589.

17. Kerrigan-Baumrind LA, Quigley HA, Pease ME, Kerrigan DF, Mitchell RS. Number of ganglion cells in glaucoma eyes compared with threshold visual field tests in the same persons. Invest Ophthalmol Vis Sci. 2000;41(3):741-748.

18. Quigley HA. Neuronal death in glaucoma. Prog Retin Eye Res. 1999;18(1):39-57.

19. Teesalu P, Airaksinen PJ, Tuulonen A. Blue-on-yellow visual field and retinal nerve fiber layer in ocular hypertension and glaucoma. Ophthalmology. 1998;105(11):20772081.

20. Mok KH, Lee VW. Nerve fiber analyzer and short-wavelength automated perimetry in glaucoma suspects: a pilot study. Ophthalmology. 2000;107(11):2101-2104.

21. Racette L, Sample PA. Short-wavelength automated perimetry. Ophthalmol Clin North Am. 2003;16(2):227236, vi-vii.

22. Bengtsson B, Heijl A. Diagnostic sensitivity of fast blueyellow and standard automated perimetry in early glaucoma: a comparison between different test programs. Ophthalmology. 2006;113(7):1092-1097.

23. Ervin JC, Lemij HG, Mills RP, Quigley HA, Thompson HW, Burgoyne CF. Clinician change detection viewing 
longitudinal stereophotographs compared to confocal scanning laser tomography in the LSU Experimental Glaucoma (LEG) Study. Ophthalmology. 2002;109(3):467481.

24. Chauhan BC, McCormick TA, Nicolela MT, LeBlanc RP. Optic disc and visual field changes in a prospective longitudinal study of patients with glaucoma: comparison of scanning laser tomography with conventional perimetry and optic disc photography. Arch Ophthalmol. 2001;119(10):1492-1499.

25. Haynes RB, Wilczynski NL. Optimal search strategies for retrieving scientifically strong studies of diagnosis from Medline: analytical survey. BMJ. 2004;328(7447):1040.

26. Deville WL, Bezemer PD, Bouter LM. Publications on diagnostic test evaluation in family medicine journals: an optimal search strategy. J Clin Epidemiol. 2000;53(1):6569.

27. Moher D, Liberati A, Tetzlaff J, Altman DG. Preferred reporting items for systematic reviews and metaanalyses: the PRISMA statement. J Clin Epidemiol. 2009;62(10):1006-1012.

28. Whiting P, Rutjes AW, Reitsma JB, Bossuyt PM, Kleijnen J. The development of QUADAS: a tool for the quality assessment of studies of diagnostic accuracy included in systematic reviews. BMC Med Res Methodol. 2003;3:25.

29. Whiting PF, Weswood ME, Rutjes AW, Reitsma JB, Bossuyt PN, Kleijnen J. Evaluation of QUADAS, a tool for the quality assessment of diagnostic accuracy studies. BMC Med Res Methodol. 2006;6:9. 